$$
\begin{aligned}
& \frac{\gamma_{\mathbf{k}}}{\omega_{p}}=-\frac{n_{1}}{n_{0}}\left(\frac{\pi}{8}\right)^{\frac{1}{2}}\left(\frac{\omega_{p}}{k v}\right)^{2}\left(\frac{\omega_{p}}{k v}-\frac{\mathbf{k} \cdot \mathbf{u}}{k v}\right) \\
& \cdot \exp \left[-\frac{1}{2}\left(\frac{\omega_{p}}{k v}-\frac{\mathbf{k} \cdot \mathbf{u}}{k v}\right)^{2}\right] .
\end{aligned}
$$

For $\left(\omega_{p}-\mathbf{k} \cdot \mathbf{u}\right)<0$ and $\left|\gamma_{1_{\mathbf{k}}} / \gamma_{0_{\mathbf{k}}}\right|>1$, waves will be unstable. From Fig. 1 for $k_{\perp}=0$ this is the region near $v_{z} \cong u$ for which $v_{z}\left(\partial f / \partial v_{z}\right)>0$, where $k_{\perp}$ is the component of $\mathbf{k}$ which is perpendicular to $\mathbf{u}$. Thus

$$
\left(\frac{\omega_{p}}{k_{\|} v}-\frac{u}{v}\right) \ll \frac{\omega_{p}}{k_{\|} v} \text { and } k_{\|} \cong \frac{\omega_{p}}{u} .
$$

To determine the range of $k_{\perp}$ for which unstable waves exist we have

$$
\begin{aligned}
& \frac{-\gamma_{1 \mathbf{k}}}{\gamma_{0 \mathrm{k}}}=-\frac{n_{1}}{n_{0}} \frac{\omega_{p}-k_{\|} u}{\omega_{p}} \\
& \cdot \exp \left\{\frac{1}{2}\left[\frac{\omega_{p}^{2}-\left(\omega_{p}-k_{\|} u\right)^{2}}{\left(k_{\|} v\right)^{2}}\right] \cos ^{2} \theta\right\},
\end{aligned}
$$

where $k_{\|}=(\mathbf{k} \cdot \mathbf{u} / u)=k \cos \theta$. We denote the $\theta=0$ growth rate by $\left|\gamma_{1_{\mathbf{k}}} / \gamma_{\mathbf{0}_{\mathbf{k}}}\right|_{\theta}=\alpha$. Since we are dealing only with very weak instabilities, we shall assume that $\alpha$ is only 2 or 3 , i.e., the growth rate due to the beam is only slightly greater than the damping rate without the beam. We then have

$$
\frac{-\gamma_{1 \mathrm{k}}}{\gamma_{0 \mathrm{k}}}=\alpha \exp \left\{-\frac{1}{2}\left[\frac{\omega_{p}^{2}-\left(\omega_{p}-k_{\|} u\right)^{2}}{\left(k_{\|} v\right)^{2}}\right] \sin ^{2} \theta\right),
$$

and the range of $k_{\perp}$ for $-\gamma_{i_{\mathrm{k}}} / \gamma_{0_{\mathrm{k}}}>1$ is given by

$$
\frac{\Delta k_{\perp}}{k_{\pi}} \cong(2 \ln \alpha)^{\frac{1}{2}} \frac{k_{\|} v}{\omega_{p}} \cong(2 \ln \alpha)^{\frac{7}{2}} k \lambda_{D}
$$

The unstable waves are thus confined to a small region in phase space of volume $\pi\left(\Delta k_{\perp}\right)^{2}\left(\Delta k_{\|}\right)$.

As discussed in reference 3 , the equilibrium spectrum to which the unstable waves grow is given by

$$
\begin{aligned}
\sum_{\mathbf{k}_{\perp}} \frac{\left|E(\mathbf{k})^{2}\right|}{8 \pi}= & \sum_{\mathbf{k}_{\perp}} \varepsilon(\mathbf{k}) \\
& =\left(\frac{L}{2 \pi}\right)^{2} \int d \mathbf{k}_{\perp} \mathcal{E}(\mathbf{k}) \cong \frac{v^{2}}{8 \pi L}\left(\frac{\omega}{k_{\|}}\right)\left(\frac{m}{e}\right)^{2} \gamma .
\end{aligned}
$$

Only those waves for which $\left(k_{\perp} / k\right) \leq\left(\Delta k_{\perp} / k\right) \cong$ $\left(k \lambda_{D}\right)$ are initially unstable, and after $e$-folding many times the spectrum is even more strongly peaked such that $k_{\perp} \leq \delta k_{\perp}=\Delta k_{\perp} /(2 R)^{\frac{1}{2}}$, where $R$ is the number of $e$-folds through which the wave amplitude has gone before reaching the equilibrium spectrum. The average value of $\mathcal{\varepsilon}(\mathbf{k})$ can thus be estimated by

$$
\begin{aligned}
\mathcal{E}(\mathbf{k}) & \cong\left(\frac{2 \pi}{L \delta k_{\perp}}\right)^{2} \frac{v^{2}}{8 \pi L} \frac{\omega}{k}\left(\frac{m}{e}\right)^{2} \gamma \\
& =\frac{8 \pi^{2} R}{\left(k \lambda_{D}\right)^{5}} \frac{\gamma}{\omega_{p}} n \lambda_{D}^{3}\left(\frac{K T}{2 L^{3}}\right) .
\end{aligned}
$$

The energy per mode in thermodynamic equilibrium is just $K T / 2 L^{3}$ and thus the wave energy, and hence the cross section is enhanced by the factor

$$
\beta=\frac{8 \pi^{2} R}{\left(k \lambda_{D}\right)^{5}}\left(\frac{\gamma}{\omega_{p}}\right) n \lambda_{D}^{3}
$$

where

$$
2 R \cong \ln \frac{8 \pi}{\left(k \lambda_{D}\right)^{5}} \frac{\gamma}{\omega_{p}} n \lambda_{D}^{3}
$$

For a typical case $\left(\gamma / \omega_{p}\right)=10^{-2}, n \lambda_{D}^{3}=10^{6}$, and $\left(k \lambda_{D}\right)=0.25$ and we obtain $R \cong 10$ and $\beta \cong 10^{10}$.

With this large enhancement of the cross section it appears possible to carry out laboratory experiments on weakly unstable plasmas. It should be mentioned that cross sections larger than equilibrium by a factor of about this magnitude have already been observed in atmospheric scattering experiments ${ }^{4}$ from the equatorial electro jet, where instabilities are likely to occur; however, the instability involved appears to be somewhat more complicated than the one discussed above.

The author would like to thank Dr. N. Rostoker and Dr. M. Rosenbluth for stimulating discussions on this topic.

This research sponsored by the Advanced $\mathrm{Re}$ search Projects Agency, Department of Defense, under project Defender, Air Force Contract No. AF29(601)-5338.

${ }^{1}$ N. Rostoker, Phys. Fluids (to be published); J. P. Dougherty and D. T. Farley, Proc. Roy. Soc. (London) A259, 79 (1960); E. E. Salpeter, Phys. Rev. 120, 1528 (1960).

2 K. L. Bowles, Phys. Rev. Letters 1, 454 (1958); National Bureau of Standards, Boulder, Colorado, Rept. 6070 (1959).

${ }^{3}$ W. E. Drummond and D. Pines, Nuclear Fusion (to be published).

${ }^{4}$ K. L. Bowles and D. T. Farley (private communication).

\section{Electric-Field Heating Threshold for Charged Particles}

\section{Anthony Demetriades}

California Institute of Technology, Pasadena, California (Received May 23, 1962; revised manuscript received June 29, 1962)

TN deriving expressions for the electrical conduc1 tivity in a gas, Kerrebrock, ${ }^{1}$ Cann, ${ }^{2}$ and others have recently recognized the importance of the elevated electron temperature to the conduction 
process. The present note attempts to point out that the role of the local electric field in maintaining the temperature difference ("nonequilibrium") between the electrons and the other species, and a threshold in the field strength below which the electronic (and ionic) mobilities are independent of field strength, can be derived from elementary considerations.

Many years ago Compton, ${ }^{3}$ assuming a billiardball collision model and a Maxwellian velocity distribution for each specie, pointed out that a charged particle drifts with a velocity proportional to the field strength $E$ at low $E$, but with velocity $v \approx E^{t}$ when the field strength is sufficiently high. Using similar ideas we derive the following formula for the drift velocity:

$$
\begin{aligned}
v=\frac{q E \lambda}{v_{T}} & \left(\frac{2}{m M}\right)^{\frac{1}{2}} \\
& \cdot\left\{1+\left[1+3 E^{2} q^{2} \lambda^{2} \frac{(m+M)^{2}}{m M^{3} v_{T}^{4}}\right]^{\frac{1}{2}}\right\}^{-\frac{1}{2}},
\end{aligned}
$$

where $q$ is the particle charge, $m$ its mass, and where $M$ and $v_{T}$ are the mass and thermal velocity of the neutral particles in which $m$ drifts; $\lambda$ is the mean free path of the charged particle in the neutral gas

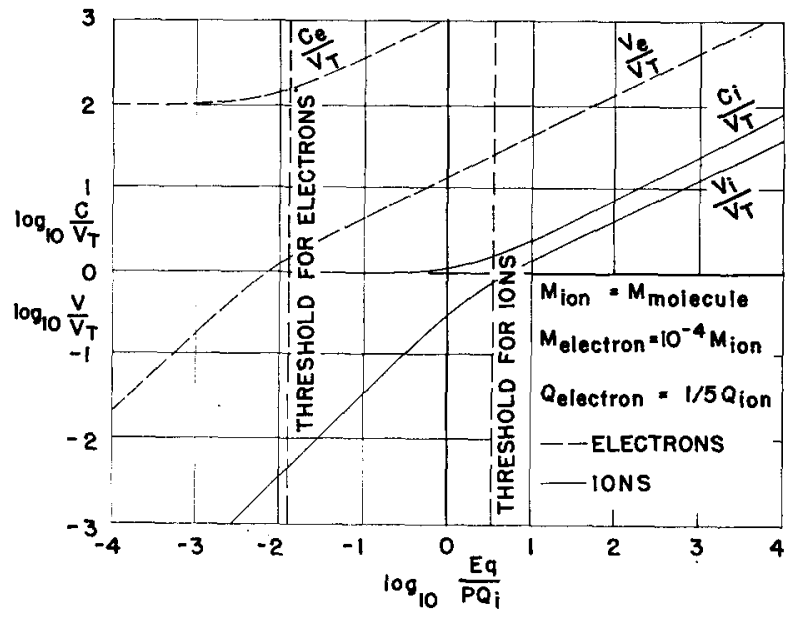

Fia. 1. Elementary mobility theory for elastic collisions of hard-sphere ions and electrons.

and is assumed independent of the collision energy. Also, the rms velocity $c$ of the charged particle is expressed by

$$
\begin{aligned}
c=v_{T}\left(\frac{M}{2 m}\right)^{t} & \\
& \left\{1+\left[1+3 E^{2} q^{2} \lambda^{2} \frac{(m+M)^{2}}{m M^{3} v_{T}^{4}}\right]^{\frac{1}{3}}\right\}^{\frac{1}{2}},
\end{aligned}
$$

such that "equilibrium" is defined by $m c^{2}=M v_{T}^{2}$. We distinguish two limiting cases. When the second term of the radical in the above bracket is much smaller than unity ("weak"-field case), the drift velocity is

$$
v=\left(q E \lambda / M v_{T}\right)(1 / \mu)^{\frac{1}{2}},
$$

with $\mu \equiv m / M$, and the charged specie equilibrates with the neutrals since $m c^{2}=M v_{T}^{2}$. When the

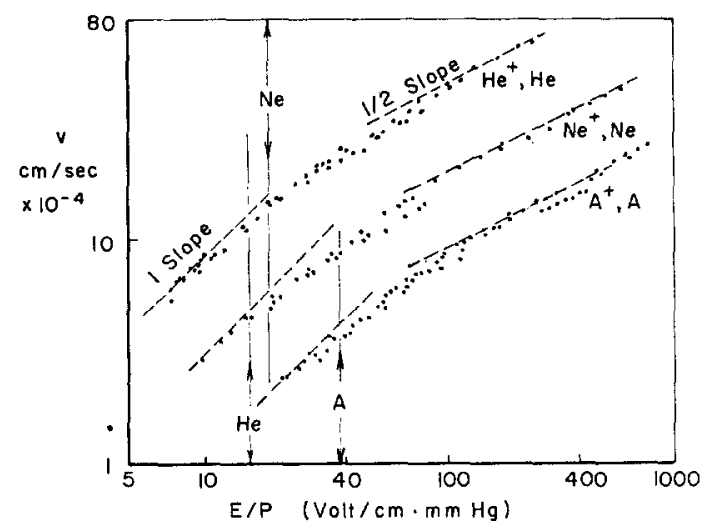

FIG. 2. Ionic drift velocity as a function of $\mathrm{E} / p$, after Hornbeck (reference 5). The critical boundaries calculated by the present theory are shown in solid vertical lines.

second term in the radical dominates, however, not only does $v$ become dependent on $E^{t}$, but $m\left(c^{2}-v^{2}\right)>M v_{T}^{2}$, generally, so that the average velocity of the charged specie begins to increase also.

If simple ideas are borrowed from kinetic theory to express $\lambda$, the threshold separating the "weak" from the "strong" field (or the "equilibrium" from the "nonequilibrium") case is given by

$$
E q / p Q=6.6\left[\mu^{\frac{1}{2}} /(\mu+1)\right],
$$

where $p$ is the actual hydrostatic pressure of the gas, and $Q$ the charged-neutral collision cross section. It is obvious that nonequilibrium is attained at much lower values of $E / p$ for electrons $\left(\mu \approx 10^{-4}-10^{-6}\right)$ than for ions $(\mu \approx 1)$. Further, very heavy ions in a very light gas should also depart from equilibrium at very low values of $E / p$, according to these ideas.

If the temperature dependence is eliminated by normalizing $v$ with $v_{T}$, the over-all role of $E / p$ can be assessed from Fig. 1, where typical values are assigned to $\mu$ and to the ratio of electron-neutral to ion-neutral cross sections. Also, using ion-neutral cross sections given by Hornbeck, ${ }^{4}$ the ion threshold is evaluated from Eq. (4) and shown on Fig. 2 superimposed on experimental data. ${ }^{4}$ The agreement is surprisingly good in view of the crudity of the assumptions underlying Eq. (4). 
The "heating" threshold for electrons occurs at extremely small values of $E / p$; if Lamb and Lin's electron-atom cross sections ${ }^{5}$ for argon are used, for example, an electron threshold of $0.8 \times 10^{-3}$ $\mathrm{V} / \mathrm{cm}$ per $\mathrm{mm} \mathrm{Hg}$ is found (versus $37 \mathrm{~V} / \mathrm{cm}$ per $\mathrm{mm} \mathrm{Hg}$ for the ion). Therefore, it is not surprising that all available data on electron drift, which are taken at above-threshold values of $E / p$, do not show a linear dependence of velocity on field strength. ${ }^{6}$

The work discussed in this paper was carried out under the sponsorship and with the financial support of the U. S. Army Research Office and the Advanced Research Projects Agency, Contract No. DA-04-495ORD-3231.

${ }^{1}$ J. I. Kerrebrock, "Conduction In Gases with Elevated Electron Temperature," Proceedings of the Second Symposium on the Engineering Aspects of Magnetohydrodynamics (Columbia University Press, New York, 1962).

2 G. L. Cann, American Rocket Society Paper Number 2396-62, Berkeley, California (1962).

${ }^{3}$ K. T. Compton, Revs. Modern Phys. 2, 210 (1930).

4 J. A. Hornbeck, Phys. Rev. 84, 615 (1951).

${ }^{5}$ L. Lamb, and S. C. Lin, J. Appl. Phys. 28, 754 (1957).

B L. L. Loeb, Basic Processes of Gaseous Electronics (University of California Press, Berkeley, California, 1955).

\section{Comments}

$O M M E N T S$ refer to papers published in The Physics of $G$ Fluids and are subject to the same length limitation as Research Notes. The Board of Editors will not hold itself responsible for the opinions expressed in the Comments.

\section{Erratum: Secondary Flow about a Sphere Rotating in a Viscous Liquid inside a Coaxially Rotating Spherical Container}

[Phys. Fluids 5, 625 (1962)]

W. L. HABERMAN

Office of Naval Research, Washington, D. C.

THE research note bearing the above title contained three typographical errors which had occurred in the author's manuscript:

(1) The expressions for $A_{1}$ and $B_{1}$ should read $-\left(\omega_{1} \lambda^{3}-\omega_{2}\right) /\left(1-\lambda^{3}\right)$ and $\left(\omega_{1}-\omega_{2}\right) R_{1}^{3} /\left(1-\lambda^{3}\right)$, respectively.

(2) The expression for $D R_{1}$ should read $D_{1}(\lambda) B_{1}^{2} R_{1}^{-3} \nu^{-1}+D_{2}(\lambda) A_{1} B_{1} \nu^{-1}$.

(3) The decimal points in the $-D_{2}(\lambda)$ column should be moved one digit to the right [e.g., at $\lambda=$ $\left.0.9,-D_{2}(\lambda)=0.3791569\right]$. 